\title{
Effect of disorder on the ground-state properties of graphene
}

\author{
R. Asgari, ${ }^{1}$ M. M. Vazifeh, ${ }^{2}$ M. R. Ramezanali, ${ }^{2}$ E. Davoudi, ${ }^{3}$ and B. Tanatar ${ }^{4}$ \\ ${ }^{1}$ School of Physics, Institute for Studies in Theoretical Physics and Mathematics, 19395-5531 Tehran, Iran \\ ${ }^{2}$ Department of Physics, Sharif University of Technology, Tehran 11155-9161, Iran \\ ${ }^{3}$ Department of Physics, Islamic Azad University, Tehran 14168-94351, Iran \\ ${ }^{4}$ Department of Physics, Bilkent University, Bilkent, 06800 Ankara, Turkey
}

(Received 22 November 2007; revised manuscript received 9 January 2008; published 27 March 2008)

\begin{abstract}
We calculate the ground-state energy of Dirac electrons in graphene in the presence of disorder. We take randomly distributed charged impurities at a fixed distance from the graphene sheet and surface fluctuations (ripples) as the main scattering mechanisms. A mode-coupling approach to the scattering rate and randomphase approximation for the ground-state energy incorporating the many-body interactions and the disorder effects yields good agreement with the experimental inverse compressibility.
\end{abstract}

DOI: 10.1103/PhysRevB.77.125432 PACS number(s): 73.63. - b, 72.10. $-\mathrm{d}, 71.55 .-\mathrm{i}, 71.10 .-\mathrm{w}$

\section{INTRODUCTION}

Two-dimensional crystals of carbon atoms (graphene) have recently been discovered. ${ }^{1}$ Graphene is a single, oneatom-thick sheet of carbon atoms arranged in a honeycomb lattice. High-quality graphene single crystals some thousands of $\mu \mathrm{m}^{2}$ in size are sufficient for most fundamental physics studies. ${ }^{2}$ There are significant efforts to grow graphene epitaxially ${ }^{3}$ by thermal decomposition of $\mathrm{SiC}$, or by vapor deposition of hydrocarbons on catalytic metallic surfaces, which could later be etched away, leaving graphene on an insulating substrate.

This stable crystal has attracted considerable attention because of its unusual effective many-body properties, ${ }^{4}$ quasiparticle properties, and Landau Fermi liquid picture, ${ }^{5}$ and the effect of electron-electron interactions on plasmon behavior and angle-resolved photoemission spectroscopy ${ }^{6}$ that follow from the chiral band states, and also because of potential applications. The low-energy quasiparticle excitations in graphene are linearly dispersing, described by Dirac cones at the edges of the first Brillouin zone. It is very hard for alien atoms to replace the carbon atoms in the graphene structure because of the robustness and specificity of the $\sigma$ bonding. Because of that, the electron mean free path $l$ in graphene can be very large. One of the important issues in graphene is its quantum transport properties; it has universal minimum conductivity at the Dirac point. Initially, it was believed that this universality is a native property ${ }^{7}$ but recent experimental ${ }^{8,9}$ and theoretical ${ }^{10-14}$ reports indicate that the transport properties are very sensitive to impurities and defects, and the minimum conductivity is not universal.

Conventional two-dimensional electron gases (2DEGs) have been a fertile source of surprising new physics for more than four decades. Although the exploration of graphene is still at an early stage, it is already clear ${ }^{7}$ that the strong-field properties of Dirac electrons in graphene are different from and as rich as those of a semiconductor heterojunction 2DEG. The Fermi liquid phenomenology of Dirac electrons in graphene $e^{5,6}$ and a conventional $2 \mathrm{DEG}^{15}$ has the same structure, since both systems are isotropic and have a single circular Fermi surface. The strength of interaction effects in a conventional 2DEG increases with decreasing carrier den- sity. At low densities, the quasiparticle weight $Z$ is small, the velocity is suppressed, ${ }^{15}$ the charge compressibility changes sign from positive to negative, ${ }^{16}$ and the spin susceptibility is strongly enhanced. ${ }^{15}$ These effects emerge from an interplay between exchange interactions and quantum fluctuations of charge and spin in the 2DEG.

In the Dirac electrons in graphene, it was shown ${ }^{4-6}$ that interaction effects also become noticeable with decreasing density, although more slowly, that the quasiparticle weight $Z$ tends to larger values, that the velocity is enhanced rather than suppressed, and that the influence of interactions on the compressibility and the spin susceptibility changes sign. These qualitative differences are due to exchange interactions between electrons near the Fermi surface and electrons in the negative energy sea, and to interband contributions to Dirac electrons from charge and spin fluctuations.

Compressibility measurements of conventional 2DEGs have been carried out, ${ }^{17}$ and it is found qualitatively that Coulomb interactions affect the compressibility at sufficiently low electron density or strong-coupling-constant region. Recently, the local compressibility of graphene has been measured ${ }^{18}$ using a scannable single-electron transistor, and it is argued that the measured compressibility is well described by the kinetic energy contribution, and it is suggested that the exchange and correlation effects have canceling contributions. From the theoretical point of view, the compressibility was first calculated by Peres et al. ${ }^{19}$ by considering the exchange contribution to the noninteracting doped or undoped graphene flake. A related quantity $\partial \mu / \partial n$ (where $\mu$ is the chemical potential and $n$ is the electron density) was recently considered by Hwang et al. ${ }^{20}$ within the same approximation. Going beyond the exchange contribution, the correlation effects were taken into account by Barlas et $a l .{ }^{4}$ based on an evaluation of graphene's exchange and random-phase approximation (RPA) correlation energies. Moreover, Sheehy and Schmalian, ${ }^{21}$ by exploiting the proximity to the relativistic electron quantum critical point, derived explicit expressions for the temperature and density dependence of the compressibility properties of graphene. All these theoretical efforts have been carried out for clean systems. Since disorder is unavoidable in any material, there has been great interest in trying to understand how disorder 
affects the physics of electrons in materials, especially here in graphene and its transport properties.

Our aim in this work is to study the ground-state properties in the presence of electron-impurity and electronelectron interactions. For this purpose, we use the selfconsistent theory of Götze ${ }^{22}$ to calculate the scattering rate, ground-state energy, and compressibility of the system at the level of the RPA including disorder effects. Our calculation is in the same spirit as our earlier work on conventional 2DEGs. ${ }^{16}$ We note that recent work of Adam et al. ${ }^{10}$ also uses a self-consistent approach, where the impurity scattering by the charge carriers is treated self-consistently in the RPA and the static conductivity is calculated in the Boltzmann kinetic theory. Thus, the main difference between the present work and that of Adam et al. ${ }^{10}$ is that we are interested in a thermodynamic quantity (compressibility), whereas the latter is aimed at calculating a transport property (conductivity). We also remark that direct solution of the Dirac equation for Dirac-like electrons incorporating the charge impurities has been discussed by Novikov $^{23}$ and the validity of the Born approximation is seriously questioned. Similar work has been carried out by Pereira et al., ${ }^{12}$ in which they studied the problem of the Coulomb charge and calculated the local density of states and the local charge by solving the Dirac equation. They found new characteristics of bound states and strong renormalization of the Van Hove singularities in the lattice description that go beyond the Dirac equation.

In this work, we consider the charged impurity and the surface roughness potentials which are established experimentally ${ }^{24,25}$ to be important. It has been demonstrated that a short-range scattering potential is irrelevant for electronic properties of graphene. ${ }^{10,26}$ We have used the same method $^{16,27}$ to investigate some properties of the conventional 2DEG. In this paper, we point out the differences between graphene and the conventional 2DEG due to disorder effects. The scattering rate behavior within our selfconsistent theory shows that impurity scattering cannot localize the carriers in graphene. The effect of disorder on spin susceptibility is similar to that on compressibility, and accordingly we will not show any results for spin susceptibility.

The rest of this paper is organized as follows. In Sec. II, we introduce the models for self-consistent calculation of the impurity effect. We then outline the calculation of compressibility. Section III contains our numerical calculations of ground state properties and comparison of models with recent experimental measurements. We conclude in Sec. IV with a brief summary.

\section{THEORETICAL MODEL}

We consider a system of 2D Dirac-like electrons interacting via the Coulomb potential $e^{2} / \varepsilon r$ and its Fourier transform $v_{q}=2 \pi e^{2} /(\varepsilon q)$, where $\varepsilon$ is the background dielectric constant. The Dirac electron gas Hamiltonian on a graphene sheet is given by

$$
\hat{\mathcal{H}}=v \sum_{\mathbf{k}, \alpha} \hat{\psi}_{\mathbf{k}, \alpha}^{\dagger}\left(\tau^{3} \otimes \sigma \cdot \mathbf{k}\right) \hat{\psi}_{\mathbf{k}, \alpha}+\frac{1}{2 A} \sum_{\mathbf{q} \neq 0} v_{q}\left(\hat{n}_{\mathbf{q}} \hat{n}_{-\mathbf{q}}-\hat{N}\right),
$$

where $v=3 t a / 2$ is the Fermi velocity, $t$ is the tight-binding hopping integral, $a$ is the spacing of the honeycomb lattice, $A$ is the sample area, and $\hat{N}$ is the total number operator. Here $\tau^{3}$ is a Pauli matrix that acts on $K$ and $K^{\prime}$, the two degenerate valleys at which the $\pi$ and $\pi^{*}$ bands touch, and $\sigma^{1}$ and $\sigma^{2}$ are Pauli matrices that act on graphene's pseudospin degrees of freedom.

A central quantity in the theoretical formulation of the many-body effects in Dirac fermions is the dynamical polarizability tensor $\chi^{(0)}(\mathbf{q}, i \Omega, \mu \neq 0)$ where $\mu$ is the chemical potential. This is defined through the one-body noninteracting Green's functions. ${ }^{28}$ The density-density response function $\chi^{(0)}(\mathbf{q}, \Omega, \mu)$ of the doped two-dimensional Dirac electron model was first consider by Shung ${ }^{29}$ as a step toward a theory of collective excitations in graphite. The Dirac electron $\chi^{(0)}(\mathbf{q}, \Omega, \mu)$ expression has been considered recently by us $^{4}$ and others. ${ }^{30}$ Implementing the Green's function $G^{(0)}(\mathbf{k}, \omega, \mu)$ in the calculation, a closed-form expression for $\chi^{(0)}(\mathbf{q}, i \Omega, \mu \neq 0)$ is found. ${ }^{4}$ To describe the properties of Dirac electrons we define a dimensionless coupling constant $\alpha_{\mathrm{gr}}=g e^{2} / v \varepsilon \hbar$, where $g=g_{v} g_{s}=4$ is the valley and spin degeneracy.

The effect of disorder is to dampen the charge-density fluctuations and results in modification of the dynamical polarizability tensor. Within the relaxation time approximation, the modified $\chi^{(0)}(\mathbf{q}, i \Omega, \mu, \Gamma)$ is given by ${ }^{31}$

$$
\chi^{(0)}(\mathbf{q}, i \Omega, \mu, \Gamma)=\frac{\chi^{(0)}(\mathbf{q}, i \Omega+i \Gamma, \mu)}{1-\frac{\Gamma}{\Omega+\Gamma}\left(1-\frac{\chi^{(0)}(\mathbf{q}, i \Omega+i \Gamma, \mu)}{\chi^{(0)}(\mathbf{q})}\right)},
$$

in which the strength of damping is represented by $\Gamma$. To include the many-body effects, we consider the densitydensity correlation function within the RPA,

$$
\chi_{\rho \rho}(\mathbf{q}, i \Omega, \mu, \Gamma)=\frac{\chi^{(0)}(\mathbf{q}, i \Omega, \mu, \Gamma)}{1-v_{q} \chi^{(0)}(\mathbf{q}, i \Omega, \mu, \Gamma)} .
$$

As the short-range disorder is shown ${ }^{10}$ to have negligible effect on the transport properties of graphene, we consider long-ranged charged impurity scattering and surface roughness as the main sources of disorder. The latter mechanism, also known as ripples, comes from either thermal fluctuations or interaction with the substrate. ${ }^{32}$ The disorderaveraged surface roughness (ripple) potential (SRP) is modeled as

$$
\left\langle\left|U_{\text {surf }}(q)\right|^{2}\right\rangle=\pi \Delta^{2} h^{2}\left(2 \pi e^{2} n / \varepsilon\right)^{2} e^{-q^{2} \Delta^{2} / 4},
$$

where $h$ and $\Delta$ are parameters describing fluctuations in the height and width, respectively. We can use the experimental results of Meyer al., ${ }^{24}$ who estimate $\Delta \sim 10 \mathrm{~nm}$ and $h \sim 0.5 \mathrm{~nm}$. It is important to point out that there are other models to take into account the surface roughness potential. The effect of bending of the graphene sheet has been studied by Kim and Castro Neto. ${ }^{33}$ This model has two main effects; first a decrease of the distance between carbon atoms, and second a rotation of the $p_{z}$ orbitals. Because of bending, the electrons are subject to a potential which depends on the structure of the graphene sheet. Another possible model is described by Katsnelson and Geim, ${ }^{26}$ considering the change of in-plane and out-of-plane displacements due to the local 
curvature of the graphene sheet. Consequently, the change of the atomic displacements results in change in nearestneighbor hopping parameters, which is equivalent to the appearance of a random gauge field described by a vector potential. These different models need to be implemented in our scheme and to be checked numerically to assess their validity in comparison to the available measurements.

The charged disorder potential (CDP) is taken to be

$$
\left\langle\left|U_{\mathrm{imp}}(q)\right|^{2}\right\rangle=n_{i} v_{q}^{2} e^{-2 q d},
$$

in which $n_{i}$ is the density of impurities and $d$ is the setback distance from the graphene sheet.

We use the mode-coupling approximation introduced by Götze $^{22}$ to express the total scattering rate in terms of the screened disorder potentials,

$$
\begin{aligned}
i \Gamma= & -\frac{v_{F} k_{F}}{2 \hbar n A} \sum_{\mathbf{q}}\left(\frac{\left\langle\left|U_{\text {imp }}(q)^{2}\right|\right\rangle}{\varepsilon^{2}(\mathbf{q})}+\frac{\left\langle\left|U_{\text {surf }}(q)\right|^{2}\right\rangle}{\varepsilon^{2}(\mathbf{q})}\right) \\
& \times \frac{\varphi_{0}(\mathbf{q}, i \Gamma)}{1+i \Gamma \varphi_{0}(\mathbf{q}, i \Gamma) / \chi^{0}(\mathbf{q})},
\end{aligned}
$$

where $\varepsilon(\mathbf{q})=1-v_{q} \chi^{(0)}(\mathbf{q})$ is the static screening function, and the relaxation function for electrons scattering from disorder is given as $\varphi_{0}(\mathbf{q}, i \Gamma)=\left[\chi^{(0)}(\mathbf{q}, i \Gamma, \mu)-\chi^{(0)}(\mathbf{q})\right] / i \Gamma$.

Since the scattering rate $\Gamma$ depends on the relaxation function $\varphi_{0}(\mathbf{q}, i \Gamma)$, which itself is determined by the response function with disorder included, the above equation needs to be solved self-consistently to yield eventually the scattering rate as a function of the coupling constant. Note that at the present level of approximation (i.e., the RPA) the static dielectric function $\varepsilon(q)$ does not depend on $\Gamma$. In the conventional 2DEG, correlation effects beyond the RPA (through the local-field factor) render $\varepsilon(q)$ also $\Gamma$ dependent. ${ }^{16}$

The ground-state energy is calculated using the coupling constant integration technique, which has the contributions $E^{\text {tot }}=E_{\text {kin }}+E_{x}+E_{c}$. The first-order "exchange" contribution per particle is given by

$$
\varepsilon_{x}=\frac{E_{x}}{N}=\frac{1}{2} \int \frac{d^{2} \mathbf{q}}{(2 \pi)^{2}} v_{q}\left(-\frac{1}{\pi n} \int_{0}^{+\infty} d \Omega \chi^{(0)}(\mathbf{q}, i \Omega, \mu, \Gamma)-1\right) .
$$

To evaluate the correlation energy in the RPA, we follow a standard strategy for uniform continuum models, ${ }^{34}$

$$
\begin{aligned}
\varepsilon_{c}^{\mathrm{RPA}}= & \frac{E_{c}}{N}=\frac{1}{2 \pi n} \int \frac{d^{2} \mathbf{q}}{(2 \pi)^{2}} \int_{0}^{+\infty} d \Omega\left\{v_{q} \chi^{(0)}(\mathbf{q}, i \Omega, \mu, \Gamma)\right. \\
& \left.+\ln \left[1-v_{q} \chi^{(0)}(\mathbf{q}, i \Omega, \mu, \Gamma)\right]\right\} .
\end{aligned}
$$

Since $\chi^{(0)}(\mathbf{q}, \Omega, \mu, \Gamma)$ is linearly proportional to $\mathbf{q}$ at large $\mathbf{q}$ and decreases only as $\omega^{-1}$ at large $\omega$, the exchange and correlation energy built by Eqs. (7) and (8) is divergent. ${ }^{4}$ In order to improve convergence, it is convenient at this point to add and subtract $\chi^{(0)}(\mathbf{q}, i \Omega, \mu=0,2 \Gamma)$ inside the frequency integral and regularize ${ }^{35}$ the exchange and correlation energy. Therefore, these ultraviolet divergences can be cured by calculating

$$
\delta \varepsilon_{x}=-\frac{1}{2 \pi n} \int \frac{d^{2} \mathbf{q}}{(2 \pi)^{2}} v_{q} \int_{0}^{+\infty} d \Omega \delta \chi^{(0)}(\mathbf{q}, i \Omega, \mu, \Gamma)
$$

and

$$
\begin{aligned}
\delta \varepsilon_{c}^{\mathrm{RPA}}= & \frac{1}{2 \pi n} \int \frac{d^{2} \mathbf{q}}{(2 \pi)^{2}} \int_{0}^{+\infty} d \Omega\left[v_{q} \delta \chi^{(0)}(\mathbf{q}, i \Omega, \mu, \Gamma)\right. \\
& \left.+\ln \left(\frac{1-v_{q} \chi^{(0)}(\mathbf{q}, i \Omega, \mu, \Gamma)}{1-v_{q} \chi^{(0)}(\mathbf{q}, i \Omega, \mu=0,2 \Gamma)}\right)\right],
\end{aligned}
$$

where $\delta \chi^{(0)}$ is the difference between the doped $(\mu \neq 0)$ and undoped $(\mu=0)$ polarizability functions. With this regularization the $q$ integrals have logarithmic ultraviolet divergences. ${ }^{4}$ We can introduce an ultraviolet cutoff for the wave vector integrals $k_{c}=\Lambda k_{F}$ which is of the order of the inverse lattice spacing and $\Lambda$ is a dimensionless quantity. The Fermi momentum is related to the density as given by $k_{F}=(4 \pi n / g)^{1 / 2}$. Once the ground state is obtained, the compressibility $\kappa$ can easily be calculated from

$$
\kappa^{-1}=n^{2} \frac{\partial^{2}\left(n \delta \varepsilon_{\mathrm{tot}}\right)}{\partial n^{2}},
$$

where the total ground-state energy is given by $\delta \varepsilon_{\text {tot }}$ $=\delta \varepsilon_{\text {kin }}+\delta \varepsilon_{x}+\delta \varepsilon_{c}^{\mathrm{RPA}}$. Here the zeroth-order kinetic contribution to the ground-state energy is $\delta \varepsilon_{\mathrm{kin}}=\frac{2}{3} \varepsilon_{F}$. We consider the dimensionless ratio $\kappa / \kappa_{0}$ where $\kappa_{0}=2 /\left(n \varepsilon_{F}\right)$ is the compressibility of the noninteracting system.

\section{NUMERICAL RESULTS}

In this section we present our calculations for groundstate properties of graphene in the presence of impurities that we model as mentioned above. The inverse compressibility $1 /\left(n^{2} \kappa\right)$ is calculated by using the theoretical models described above and the results are compared with the recent experimental measurements. In all numerical calculations we consider $d=0.5 \mathrm{~nm}$. The electron density is taken to be $1 \times 10^{12} \mathrm{~cm}^{-2}$ for Figs. 1-3.

Increasing disorder (increasing $n_{i}$ or decreasing $d$ for a charge disorder potential or increasing $h$ for a surface roughness potential) decreases $\chi^{(0)}(q, \Omega, \mu, \Gamma)$ as the scattering rate $\Gamma$ gets bigger. Thus, decreasing $\chi^{(0)}(q, \Omega, \mu, \Gamma)$ (or increasing correlation effects) results in a stronger disorder potential. Although $\Gamma$ increases with increasing $\alpha_{\mathrm{gr}}$, apparently it grows to a saturation limit and does not diverge. This behavior is quite different from what is seen in a conventional $2 \mathrm{DEG},{ }^{16}$ when the many-body effects influence the scattering rate through the local-field factor. In the conventional 2DEG system, at a critical level of disorder this nonlinear feedback causes $\Gamma$ to increase rapidly and diverge, which is taken as an indication of the localization of carriers. However, in graphene, our calculations show that $\Gamma$ does not diverge; therefore impurities cannot localize carriers and we have a weakly localized system in the presence of impurities, compatible with experimental observations. ${ }^{36}$ We can understand the saturated behavior of $\Gamma$ qualitatively as follows. In the context of a conventional 2DEG, the Mott argument says 


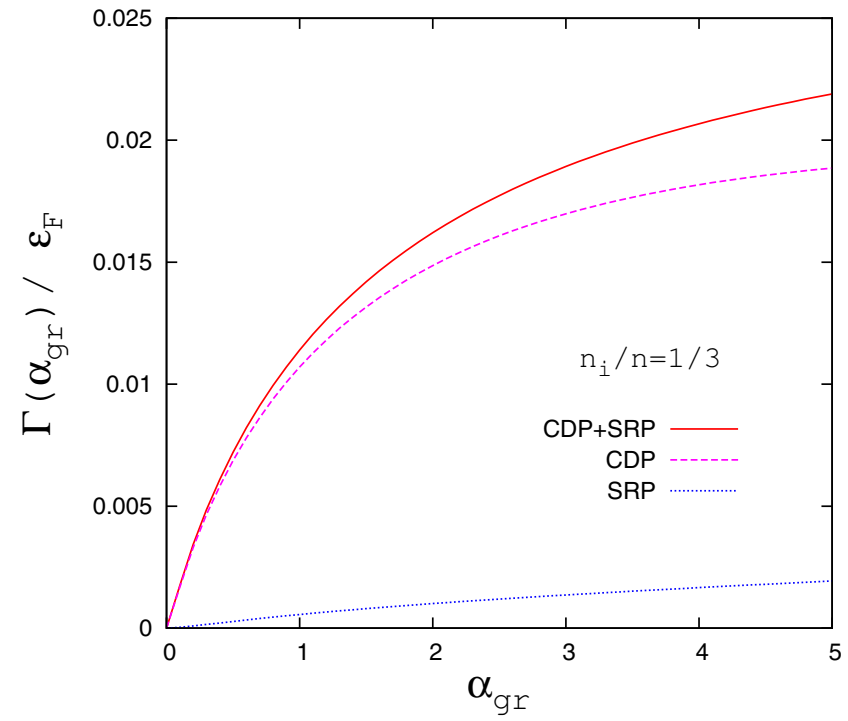

FIG. 1. (Color online) Scattering rate $\Gamma$ as a function of the coupling constant $S$ for both the charge disorder potential (CDP) and surface roughness potential (SRP) contributions.

that the mean free path $l$ in a metal cannot be shorter than the wavelength $\lambda$. Since $l$ is proportional to the inverse of $\Gamma$, for large values of $\Gamma$ obtained in a $2 \mathrm{DEG}$, the electron mean free path decreases and becomes less than or equal to $\lambda$. At this point we should have a metal-insulator phase transition. In the context of graphene, on the other hand, Mott's argument suggests that the light is unaffected by any roughness (one source of scattering) on a scale shorter than its wavelength. Consequently there is a lower limit for the electron's mean free path in graphene, and it turns out that we have a maximum (saturation) value for $\Gamma$.

The issue of localization in graphene has recently attracted some attention and the chiral nature of the electron behavior has been discussed in the literature. ${ }^{37,38}$ Suzuura and $\mathrm{Ando}^{37}$ claimed that the quantum correction to the conductivity in graphene can differ from what is observed in a normal 2DEG because the elastic scattering in graphene can possibly change the sign of the localization correction and turn weak localization into weak antilocalization for the region where the intervalley scattering time is much larger than the phase coherence time. Further consideration of the behavior of the quantum correction to the conductivity in graphene $^{38}$ concluded that this behavior is entirely suppressed due to time-reversal-symmetry breaking of electronic states around each degenerate valley.

We have found through our calculations that $\Gamma$ increases with increasing $n_{i} / n$ as a function of $\alpha_{g r}$. Figure 1 shows $\Gamma$ for various scattering mechanisms. It is clear that the CDP is the dominant mechanism for $\Gamma$ in our model. The effect of the SRP is mostly negligible, except at large values of the coupling constant. This finding is to be contrasted with the statement of Martin et al. ${ }^{18}$ that both substrate-induced structural distortions (SRP) and chemical doping (CDP) are conceivable sources of density fluctuations. We stress that our model calculations indicate that at realistic coupling constant values (see Fig. 1) only the charged impurity scattering dominates.
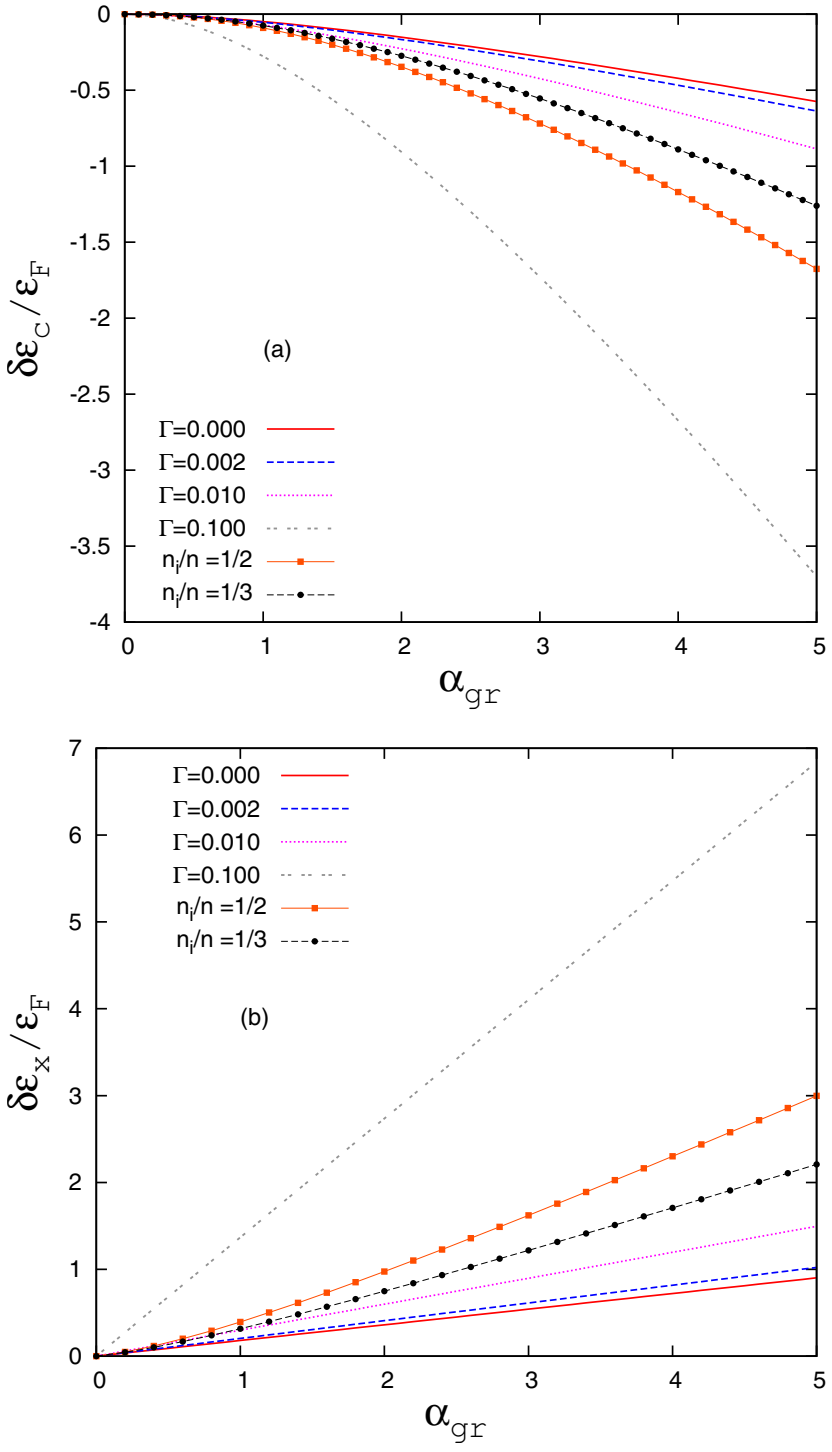

FIG. 2. (Color online) (a) Correlation energy $\delta \varepsilon_{c}$ as a function of the coupling constant $\alpha_{\text {gr }}$ for cutoff value $\Lambda=k_{c} / k_{F}=50$. (b) Exchange energy $\delta \varepsilon_{x}$ as a function of the coupling constant $\alpha_{\mathrm{gr}}$ for cutoff value $\Lambda=50$. Results of fixed $\Gamma$ values are compared to those calculated within the mode-coupling approximation.

We have calculated the exchange and correlation energies as a function of $\alpha_{\mathrm{gr}}$ in the presence of disorder. It is found that the disorder effects become more appreciable at large coupling constants, within the mode-coupling approximation. The exchange energy is positive ${ }^{4}$ because our regularization procedure implicitly selects the chemical potential of undoped graphene as the zero of energy; doping either occupies quasiparticle states with positive energies or empties quasiparticles with negative energies. Figure 2(a) shows the correlation energy $\delta \varepsilon_{c}$ as a function of $\alpha_{\mathrm{gr}}$. It appears that the disorder effects become more appreciable at large coupling constant. Note that $\delta \varepsilon_{c}$ has the same density dependence as $\delta \varepsilon_{x}$ apart from the weak dependence on $\Lambda$. In contrast to the exchange energy, Fig. 2(b), the correlation energy is negative. ${ }^{4}$ Figure 3 shows the charge compressibility $\kappa / \kappa_{0}$ scaled by its noninteracting contribution as a function of $\alpha_{\mathrm{gr}}$ for various models of $\Gamma$. The behavior of $\kappa$ shows some 


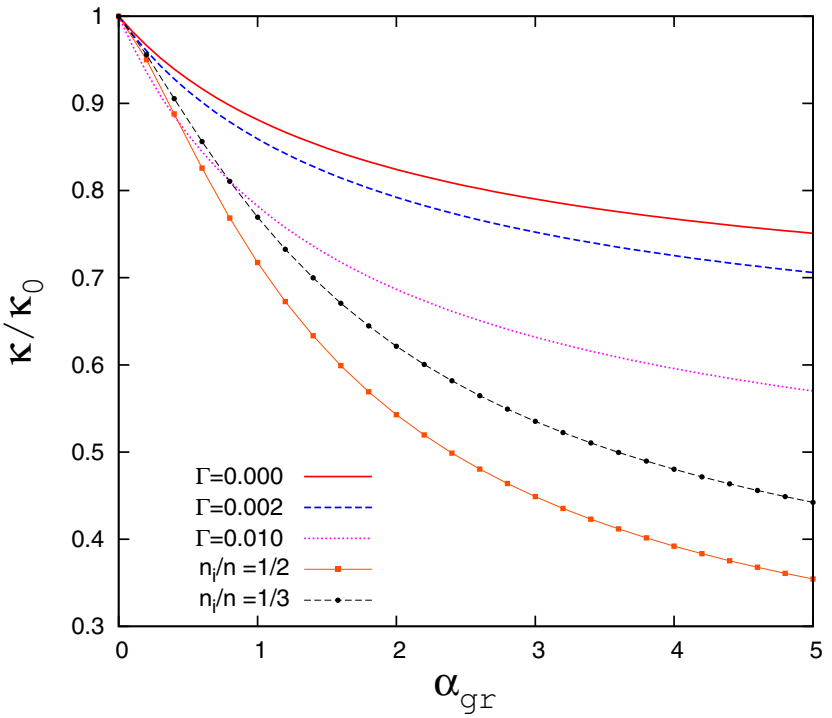

FIG. 3. (Color online) Compressibility $\kappa / \kappa_{0}$ scaled by that of a noninteracting clean system as a function of the coupling constant $\alpha_{\text {gr }}$ for cutoff value $\Lambda=50$.

novel physics, which is qualitatively different from the physics known in the conventional 2DEG. Exchange makes a positive contribution to the inverse compressibility and thus tends to reduce (rather than enhance) the compressibility. On the other hand, correlations make a negative contribution to the inverse compressibility and thus tend to enhance $\kappa$. In the conventional 2DEG both contributions tend to enhance the compressibility. In the case of graphene instead, apparently exchange and correlation compete with each other ${ }^{18}$ in determining the compressibility of the system. It is interesting to note that similar physics is true also in the spin susceptibility. ${ }^{4}$

In Fig. 4 we compare our theoretical predictions for the inverse compressibility of doped graphene with the experimental results of Martin et al. ${ }^{18}$ For definiteness we take $\Lambda=k_{c} / k_{F}$ to be such that $\pi\left(\Lambda k_{F}\right)^{2}=(2 \pi)^{2} / \mathcal{A}_{0}$, where $\mathcal{A}_{0}=3 \sqrt{3} a_{0}^{2} / 2$ is the area of the unit cell in the honeycomb lattice, with $a_{0} \simeq 1.42 \AA$ the carbon-carbon distance. With this choice $\Lambda \simeq\left(g n^{-1} \sqrt{3} / 9.09\right)^{1 / 2} \times 10^{2}$, where $n$ is the electron density in units of $10^{12} \mathrm{~cm}^{-2}$. Martin et al. ${ }^{18}$ fitted the experimental inverse compressibility $\left(n^{2} \kappa\right)^{-1}$ to the kinetic term using a single-parameter Fermi velocity which is larger than the bare Fermi velocity. Note that the kinetic term in graphene has the same density dependence as the leading exchange and correlation terms.

As is clear in Fig. 4 the inverse compressibility of a noninteracting system is below the experimental data. By increasing the interaction effects, i.e., increasing the coupling constant strength $\alpha_{\mathrm{gr}}$, our theoretical results move up. Unfortunately, in the experimental sample, the value of $\alpha_{\mathrm{gr}}$ is not specified and we considered it to be $\approx 1$. Therefore, including the exchange-correlation effects in our RPA theory gives results very close to the experimental data. Furthermore, the results of incorporating the impurity density $n_{i}=10^{10} \mathrm{~cm}^{-2}$ in the system and solving the self-consistent equations to obtain the scattering rate value yield very good agreement with the measured values in the large- and mid-electron-

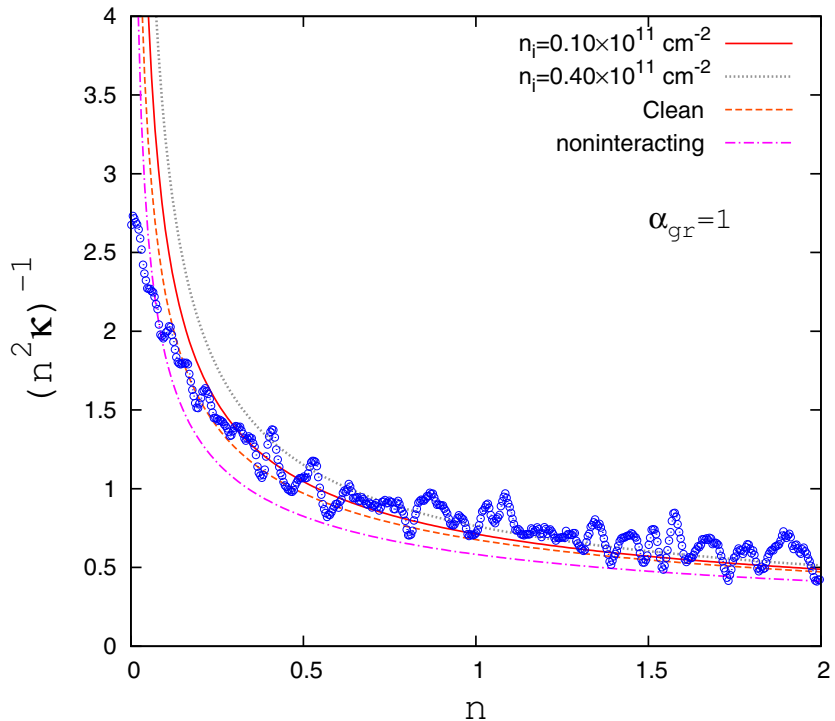

FIG. 4. (Color online) Inverse compressibility $\left(n^{2} \kappa\right)^{-1}=\partial \mu / \partial n$ [in units of $\mathrm{meV}\left(10^{-10} \mathrm{~cm}^{-2}\right)$ ] as a function of the electron density (in units of $10^{12} \mathrm{~cm}^{-2}$ ). The filled squares are the experimental data of Martin et al. (Ref. 18).

density regions. We have examined the inverse compressibility using the kinetic term contribution only, including a fitting value for the Fermi velocity, and our numerical results are well described by a fitting velocity about $1.28 v_{F}$. We would stress here that this fitting velocity is different from the renormalized velocity defined within the Landau Fermi liquid theory in graphene. ${ }^{5}$

In a recent calculation of $\partial \mu / \partial n$ within the Hartree-Fock approximation in grapheme, where $\mu$ is the chemical potential and $n$ is the electron density, Hwang et al. ${ }^{20}$ stated that correlation and disorder effects would introduce only small corrections. This is not true in general, since it has been shown by Barlas et al. ${ }^{4}$ that the correlation effects are essential in the ground-state properties. Although these effects are not significant in the regime of very weak interaction strength and high electron density, the inclusion of manybody exchange-correlation effects together with the disorder effect are necessary to get agreement with quantities measured in the experiments of Martin et al. ${ }^{18}$ It would be useful to carry out further experimental work at larger interaction strengths to assess the role played by correlation effects.

\section{CONCLUSION}

We have studied the ground-state thermodynamic properties of a graphene sheet within the random-phase approximation, incorporating the impurities in the system. Our approach is based on a self-consistent calculation including impurity effects and many-body electron-electron interactions. We have used a model surface roughness potential together with the charged disorder potential in the system. Our calculations of inverse compressibility, when compared with recent experimental results of Martin et al., ${ }^{18}$ demonstrate the importance of including correlation effects together with disorder effects correctly in the thermodynamic quantities. 
We remark that, in the very low-density region, the system is highly inhomogeneous; here the experimental data tend to a constant and the effect of the impurities is very important. A model going beyond the RPA is necessary to account for increasing correlation effects at low density. To describe the experimental data in this region, more sophisticated theoretical methods incorporating inhomogeneities are needed. One approach would be the density-functional theory where Dirac electrons in the presence of impurities are considered.

\section{ACKNOWLEDGMENTS}

We thank J. Martin for providing us with experimental data and M. Polini for useful discussions. B.T. is supported by TUBITAK (Grant No. 106T052) and TUBA.
${ }^{1}$ K. S. Novoselov, A. K. Geim, S. V. Morozov, D. Jiang, Y. Zhang, S. V. Dubonos, I. V. Grigorieva, and A. A. Firsov, Science 306, 666 (2004).

${ }^{2}$ A. K. Geim and K. S. Novoselov, Nat. Mater. 6, 183 (2007).

${ }^{3}$ C. Berger, Z. Song, X. Li, X. Wu, N. Brown, C. Naud, D. Mayou, T. Li, J. Hass, A. N. Marchenkov, E. H. Conrad, P. N. First, and W. A. de Heer, Science 312, 1191 (2006).

${ }^{4}$ Y. Barlas, T. Pereg-Barnea, M. Polini, R. Asgari, and A. H. MacDonald, Phys. Rev. Lett. 98, 236601 (2007).

${ }^{5}$ M. Polini, R. Asgari, Y. Barlas, T. Pereg-Barnea, and A. H. MacDonald, Solid State Commun. 143, 58 (2007).

${ }^{6}$ M. Polini, R. Asgari, G. Borghi, Y. Barlas, T. Pereg-Barnea, and A.H. MacDonald, Phys. Rev. B 77, 081411(R) (2008).

${ }^{7}$ K. S. Novoselov, A. K. Geim, S. V. Morozov, D. Jiang, M. I. Katsnelson, I. V. Grigorieva, S. V. Dubonos, and A. A. Firsov, Nature (London) 438, 197 (2005).

${ }^{8}$ Y.-W. Tan, Y. Zhang, K. Bolotin, Y. Zhao, S. Adam, E. H. Hwang, S. Das Sarma, H. L. Stormer, and P. Kim, Phys. Rev. Lett. 99, 246803 (2007).

${ }^{9}$ S. Cho and M. S. Fuhrer, arXiv:0705.3239 (unpublished).

${ }^{10}$ S. Adam, E. H. Hwang, and S. Das Sarma, arXiv:0708.0404, Physica E (to be published); S. Adam, E. H. Hwang, V. H. Galitski, and S. Das Sarma, Proc. Natl. Acad. Sci. U.S.A. 104, 18392 (2007)

${ }^{11}$ T. Ando, J. Phys. Soc. Jpn. 75, 074716 (2006); R. R. Biswas, S. Sachdev, and D. T. Son, Phys. Rev. B 76, 205122 (2007).

${ }^{12}$ V. M. Pereira, J. Nilsson, and A. H. Castro Neto, Phys. Rev. Lett. 99, 166802 (2007).

${ }^{13}$ M. I. Katsnelson, Mater. Today 10, 20 (2007).

${ }^{14}$ A.H. Castro Neto, F. Guinea, and N.M.R. Peres, Phys. World 19, 33 (2006).

${ }^{15}$ R. Asgari and B. Tanatar, Phys. Rev. B 74, 075301 (2006); R. Asgari, B. Davoudi, M. Polini, G. F. Giuliani, M. P. Tosi, and G. Vignale, ibid. 71, 045323 (2005).

${ }^{16}$ R. Asgari and B. Tanatar, Phys. Rev. B 65, 085311 (2002).

${ }^{17}$ J. P. Eisenstein, L. N. Pfeiffer, and K. W. West, Phys. Rev. B 50, 1760 (1994); I. S. Millard, N. K. Patel, C. L. Foden, E. H. Linfield, M. Y. Simmons, D. A. Ritchie, and M. Pepper, ibid. 55, 6715 (1997).

${ }^{18}$ J. Martin, N. Akerman, G. Ulbricht, T. Lohmann, J. H. Smet, K. von Klitzing, and A. Yacoby, Nat. Phys. 4, 144 (2007).

${ }^{19}$ N. M. R. Peres, F. Guinea, and A. H. Castro Neto, Phys. Rev. B 72, 174406 (2005).

${ }^{20}$ E. H. Hwang, Ben Yu-Kuang Hu, and S. Das Sarma, Phys. Rev.
Lett. 99, 226801 (2007).

${ }^{21}$ D. E. Sheehy and J. Schmalian, Phys. Rev. Lett. 99, 226803 (2007).

${ }^{22}$ W. Götze, Solid State Commun. 27, 1393 (1978); Philos. Mag. B 43, 219 (1981).

${ }^{23}$ D. Novikov, Appl. Phys. Lett. 91, 102102 (2007); Phys. Rev. B 76, 245435 (2007).

${ }^{24}$ J. C. Meyer, A. K. Geim, M. I. Katsnelson, K. S. Novoselov, T. J. Booth, and S. Roth, Nature (London) 446, 60 (2007).

${ }^{25}$ M. Ishigami, J. H. Chen, W. G. Cullen, M. S. Fuhrer, and E. D. Williams, Nano Lett. 7, 1643 (2007).

${ }^{26}$ M. I. Katsnelson and A. K. Geim, Philos. Trans. R. Soc. London, Ser. A 366, 195 (2008).

${ }^{27}$ R. Asgari, B. Davoudi, and B. Tanatar, Solid State Commun. 130, 13 (2004).

${ }^{28}$ J. González, F. Guinea, and M. A. H. Vozmediano, Nucl. Phys. B 424, 595 (1994).

${ }^{29}$ Kenneth W.-K. Shung, Phys. Rev. B 34, 979 (1986).

${ }^{30}$ B. Wunsch, T. Stauber, F. Sols, and F. Guinea, New J. Phys. 8, 318 (2006); X.-F. Wang and T. Chakraborty, Phys. Rev. B 75, 041404(R) (2007); E. H. Hwang and S. Das Sarma, ibid. 75, 205418 (2007).

${ }^{31}$ N. D. Mermin, Phys. Rev. B 1, 2362 (1970).

${ }^{32}$ A. Fasolino, J.H. Los, and M. I. Katsnelson, Nat. Mater. 6, 858 (2007); N. Abedpour, M. Neek-Amal, R. Asgari, F. Shahbazi, N. Nafari, and M. R. Tabar, Phys. Rev. B 76, 195407 (2007).

${ }^{33}$ E.-A. Kim and A. H. Castro Neto, arXiv:cond-mat/0702562 (unpublished).

${ }^{34}$ G. F. Giuliani and G. Vignale, Quantum Theory of the Electron Liquid (Cambridge University Press, Cambridge, U.K., 2005).

${ }^{35}$ The local chemical potential is changed infinitesimally due to the impurity effect and therefore in the case of undoped graphene we have small $\delta \mu$ around the Dirac point. In this case, scattering processes occur in both conduction and valance bands near the Dirac point, and the rate of change of the one-body density matrix due to collisions is approximated by $(d \rho / d t)_{\text {coll }}=-\gamma(\rho$ $\left.-\rho_{0}^{c}\right)-\gamma\left(\rho-\rho_{0}^{v}\right)$. We assume that $\rho_{0}^{c}=\rho_{0}^{v}$.

${ }^{36}$ S. V. Morozov, K. S. Novoselov, M. I. Katsnelson, F. Schedin, L. A. Ponomarenko, D. Jiang, and A. K. Geim, Phys. Rev. Lett. 97, 016801 (2006).

${ }^{37}$ H. Suzuura and T. Ando, Phys. Rev. Lett. 89, 266603 (2002).

${ }^{38}$ E. McCann, K. Kechedzhi, V. I. Fal'ko, H. Suzuura, T. Ando, and B. L. Altshuler, Phys. Rev. Lett. 97, 146805 (2006); A. F. Morpurgo and F. Guinea, ibid. 97, 196804 (2006). 